(2) Open Access Full Text Article

\title{
Hypoxia: from basic mechanisms to therapeutics - a meeting report on the Keystone and HypoxiaNet Symposium
}

This article was published in the following Dove Press journal:

Hypoxia

23 October 2015

Number of times this article has been viewed

Karen A Nolan

Carsten C Scholz

Institute of Physiology, University of Zürich, Zürich, Switzerland
Correspondence: Carsten C Scholz Institute of Physiology, University of Zürich, Winterthurerstrasse 190, 8057 Zürich, Switzerland Tel +4I 446355075

Email carsten.scholz@uzh.ch
Abstract: In May 2015, the hypoxia research community came together at the largest meeting in this field to date, to present and discuss their most recent and mainly unpublished findings. This meeting report aims to summarize the data presented at this conference, which were broadly separated into the areas of the cellular hypoxic response, the relevance of the hypoxic response in health and disease, and the development of new therapeutics targeting the hypoxic response.

Keywords: hydroxylase, HIF, PHD, FIH, oxygen, hydroxylase inhibitor

\section{Introduction}

The Keystone Symposium "Hypoxia: From Basic Mechanisms to Therapeutics" was held in Dublin, Ireland, from May 12th-17th, 2015 in conjunction with the European HypoxiaNet meeting. The meeting was organized by four internationally highly regarded experts from the hypoxia field: Dr Cormac T Taylor (University College Dublin, Dublin, Ireland), Dr M Celeste Simon (The Howard Hughes Medical Institute (HHMI)/University of Pennsylvania, Philadelphia, PA, USA), Dr Sean P Colgan (University of Colorado Denver, Denver, CO, USA), and Dr Roland H Wenger (University of Zürich, Zürich, Switzerland).

HypoxiaNet (http://www.hypoxianet.com) is a network of over 600 European researchers in the hypoxia field from 28 different countries that was established by Dr Roland H Wenger and Dr Dörthe M Katschinski (University of Göttingen, Göttingen, Germany) in 2009. The initial funding for HypoxiaNet was provided by the European Cooperation in Science and Technology (COST) Action program of the EU, and this joint symposium was the 11th HypoxiaNet meeting to be held since 2009.

This conference was also the seventh Keystone meeting in the hypoxia research field and the first occasion on which it was held outside of North America. ${ }^{1}$ It was a huge success, with almost 500 participants from 36 different countries including over $200 \mathrm{PhD}$ students and postdoctoral fellows, making it the largest gathering of hypoxiafocused researchers that has taken place to date.

Hypoxia, or a decreased oxygen supply over demand, plays a major role in health and disease. ${ }^{2-6}$ Changes in whole-body oxygen supply are sensed in the carotid body and in the kidney, leading to the adaptation of alveolar ventilation and of erythropoietin (Epo) synthesis, respectively. ${ }^{7}$ On the cellular level, decreased oxygen availability is sensed ubiquitously by four different oxygen-sensing hydroxylases (prolyl hydroxylase domains [PHDs] 1-3 and factor-inhibiting hypoxia-inducible factor [HIF] [FIH]). The hydroxylases were discovered as regulators of HIF, the master transcription factor for cellular 
adaptation to hypoxic conditions. ${ }^{8-12,15}$ Hypoxia-sensitive signaling pathways play a major role in disease, ranging from anemia and cancer to chronic inflammation. ${ }^{13,14}$ Many pharmaceutical companies are currently developing inhibitors of the oxygen-sensing enzymes, with compounds now in clinical phase IIb and phase III trials. ${ }^{16}$ All these aspects, as well as HIF-independent aspects of the hypoxic response, were presented and discussed during the Keystone and HypoxiaNet Symposium.

The meeting commenced with a keynote address by Dr Peter F Carmeliet (University of Leuven, VIB Vesalius Research Centre, Leuven, Flanders, Belgium), who highlighted the important and diverse roles of oxygen sensing in spine formation, metastasis, and neuroprotection. Furthermore, he described the importance of endothelial cell metabolism in angiogenesis.

During several sessions, distinguished scientists presented their latest (and mostly unpublished) data from research in the hypoxia field. In addition, each session contained short talks that gave young investigators the opportunity to present their data to the wider hypoxia research community. Due to the size of the conference and the diversity of the field, it was not possible to provide a comprehensive summary of all topics discussed at the meeting in this short meeting report. We focused instead on giving an overview of the meeting to highlight the current interests and focus of the field, with some examples in each case. The topics discussed at this meeting have been broadly separated into the following three sections: 1) "Unraveling the cellular hypoxic response", 2) "Relevance and impact of the hypoxic response in health and disease", and 3) "Developing new therapeutics targeting the hypoxic response".

\section{Unraveling the cellular hypoxic response}

The discovery of HIF was the first major step toward the unraveling of the cellular hypoxic response. ${ }^{17}$ This discovery was followed by the description of the cellular oxygen sensors PHDs 1-3 and FIH ${ }^{8-12,15}$ a decade later. Since then, the cellular hypoxic response has been extensively studied, with the major focus on the HIF transcription factor and on the oxygen-sensing hydroxylases. Three major areas about the cellular hypoxic response were discussed at the Keystone and HypoxiaNet Symposium.

\section{The search for novel cellular oxygen sensors}

The HIF hydroxylases belong to the Fe(II)- and 2-oxoglutaratedependent dioxygenase superfamily, which consists of approximately 70 different proteins. Therefore, it seems likely that other 2-oxoglutarate-dependent oxygen sensors are present in cells. Also, existence of proteins with oxygen sensing capability from other protein families cannot be excluded. However, to date no further oxygen sensor has been described at the level of detail that PHDs 1-3 and FIH have been described at. Interestingly, Dr Peter J Ratcliffe (University of Oxford, Oxford, UK) presented data describing a possible new oxygen-sensing enzyme, which is surprisingly a monooxygenase and may be involved in oxygen-dependent neuropeptide processing and modification.

\section{Hydroxylase targets outside the HIF pathway}

To date, only few proteins outside the HIF pathway have been found to be regulated by proline hydroxylation. ${ }^{18-21}$ FIH-dependent asparagine hydroxylation has been described for several proteins containing ankyrin-repeat domains, such as ASPP2, I $\mathrm{B} \alpha$, notch-1, and tankyrase. ${ }^{22-25}$ However, the functional impact of asparagine hydroxylation outside the HIF pathway is less clear. Dr Daniel J Peet (University of Adelaide, Adelaide, SA, Australia) presented results demonstrating that FIH-dependent asparagine hydroxylation of the ion channel transient receptor potential vanilloid 3 leads to the oxygen-dependent regulation of its activity. Dr Cormac T Taylor demonstrated that a specific deubiquitinating enzyme may be a new FIH target protein and that FIH-dependent asparagine hydroxylation of this deubiquitinase may result in changes in cellular energy metabolism. This could give a first mechanistic insight into the previously reported metabolic phenotype of FIH knockout mice. ${ }^{26}$ Dr Dörthe M Katschinski discussed her data showing that hypoxia leads to increased clathrin- and caveolin-independent endocytosis and to changes between the cis and trans Golgi network. This regulation seemed to be independent of HIF as well.

\section{Novel regulatory mechanisms of the HIF transcription factor}

It has been demonstrated that HIF-1 $\alpha$ is not only regulated by the hydroxylases but is also regulated by several other mechanisms. ${ }^{27}$ For example, activation of the NF- $\kappa B$ pathway can lead to an upregulation of the HIF- $1 \alpha$ protein through enhanced transcription of the HIF $1 \mathrm{~A}$ gene. ${ }^{28-30}$ Another example is the regulation of HIF-1 $\alpha$ by the microRNA miR-155, which serves as a negative feedback loop in response to hypoxia. ${ }^{31}$ Dr Edurne Berra (Center for Cooperative Research in Biosciences [CIC 
bioGUNE], Biscay, Spain) highlighted a role for deubiquitinating enzymes in the regulation of HIF- $1 \alpha$ protein levels and discussed a specific deubiquitinase as a newly discovered regulator of HIF-1 $\alpha$. Dr Thomas Kietzmann (University of Oulu, Oulu, Finland) presented data about a novel regulation of HIF-1 $\alpha$ by an E3 ubiquitin ligase in conjunction with a specific deubiquitinase. Dr Paul N Moynagh (National University of Ireland, Maynooth, Ireland) discussed a novel impact of a further specific E3 ubiquitin ligase on $\mathrm{HIF}-1 \alpha$ regulating IL- $1 \beta$ expression. Overall, these presentations illustrate that the interaction of the HIF pathway and the ubiquitin system is a current and rapidly developing focus of the field.

\section{Relevance and impact of the hypoxic response in health and disease}

Reduced oxygen availability leads to intrinsic physiologic responses at both cellular and systemic levels. Our ability to respond to a decrease in oxygen availability is imperative for adaptation and survival. Hypoxia is associated with most diseases both acute and chronic. ${ }^{32}$ Not surprisingly, pathologic conditions can result in altered/impaired physiologic responses to hypoxia. ${ }^{33}$ Unraveling the complex nature of oxygen sensing in health and disease states is essential for further understanding of disease processes as well as the identification of potential therapeutic targets. Three particular areas of interest were highlighted at the Keystone and HypoxiaNet Symposium.

\section{Physiological oxygen sensing by the kidney}

Discrepancy in findings identifying Epo-producing cells in the kidney has led historically to debate over the exact identity and location of these cells, although recent results point to renal cortical interstitial cells. In addition, the detailed cellular oxygen sensing mechanisms of the Epo-producing cells of the kidney remain to be fully elucidated due to the absence of a cell culture system of renal Epo-producing cells. ${ }^{34}$ Dr Roland H Wenger and Dr Volker H Haase (Vanderbilt University, Nashville, TN, USA) presented different mouse models designed for the investigation of these cells in vivo and discussed possibilities for future research in order to further elucidate oxygen sensing mechanisms in the kidney. Dr Josef Prchal (University of Utah, Salt Lake City, UT, USA) discussed a link between renal oxygen sensing and the adaptation of the Tibetan population to high altitude by presenting mutations in the hypoxia-sensing pathways that were discovered in the Tibetan population and that lead to polycythemia.

\section{Mechanisms of oxygen sensing by the carotid body}

The carotid body plays an essential role in the physiologic response to reduced oxygen availability by adapting alveolar ventilation. ${ }^{35}$ However, the exact molecular mechanism(s) of oxygen sensing in the carotid body remain(s) to be elucidated. ${ }^{36}$ Dr José López-Barneo (Hospital Universitario Virgen del Rocío, Seville, Spain) argued that reactive oxygen species are important intracellular messengers for sensing of low oxygen concentrations, while Dr Nanduri R Prabhakar (University of Chicago, Chicago, IL, USA) discussed $\mathrm{H}_{2} \mathrm{~S}$, $\mathrm{CO}$, and $\mathrm{NO}$ as relevant gaseous messengers.

\section{The hypoxic response in cancer}

The impact of the hypoxic response in the complex setting of tumorigenesis is a key area of interest. ${ }^{37}$ Hypoxia is in many tumors a negative prognostic and predictive factor due to its impact on a broad range of cellular and physiological/pathophysiological responses including angiogenesis, vasculogenesis, invasiveness, metastasis, metabolism, cell death resistance, genomic instability, and chemo- and radioresistance. ${ }^{38}$ The role of the oxygen-sensing pathway in cancer is likely to be highly context-dependent. ${ }^{39,40}$ At the Keystone and HypoxiaNet Symposium, Dr Till Acker (University of Giessen, Giessen, Germany) focused on the role of PHD 3 in cancer and demonstrated that loss of PHD 3 promotes tumor growth and impacts on therapy response. Dr Gregg L Semenza (John Hopkins University School of Medicine, Baltimore, MD, USA) discussed the role of HIF-1 $\alpha$ and the transcriptional co-activator with PDZ-binding motif (TAZ) in the breast cancer stem cell phenotype and their impact on breast cancer stem cell enrichment. In clear-cell renal cell carcinoma, HIF-2 $\alpha$ was found to promote tumor cell viability through regulation of lipid storage, as presented by Dr M Celeste Simon.

\section{Developing new therapeutics targeting the hypoxic response}

The development of pharmaceutical drugs targeting the HIF pathway is a highly dynamic, rapidly growing, and promising field. To date, 12 different companies are known to have developed inhibitors targeting the oxygen-sensing hydroxylases: FibroGen, Bayer Pharma AG, GlaxoSmithKline, Akebia Therapeutics, Amgen, Merck \& Co, Inc., Janssen, Akros Pharma Inc., Procter and Gamble, Daiichi Sankyo Inc., Crys- 
tal Genomics, and Kowa. ${ }^{16,41}$ In addition, Astellas Pharma and AstraZeneca collaborate with FibroGen. ${ }^{16}$ At the beginning of this year 26 different clinical trials with hydroxylase inhibitors were registered with http://www.ClinicalTrials. gov. ${ }^{16}$ Current clinical trials and emerging therapeutic options for hydroxylase inhibitors were discussed at this meeting.

\section{Development of hydroxylase inhibitors for treatment of anemia}

Several different hydroxylase inhibitors are currently investigated in clinical trials for their efficacy in the treatment of anaemia in chronic kidney disease. The pharmaceutical companies FibroGen, GlaxoSmithKline, and Bayer Pharma AG presented their most recent advances in clinical trials at this meeting. Importantly, Phase III clinical trials are currently starting for the FibroGen compound FG-4592 (ASP1517, Roxadustat). The different compounds in several clinical trial phases have recently been reviewed. ${ }^{16}$

\section{Emerging treatment options for hydroxylase inhibitors}

To date, one highly promising effect of hydroxylase inhibitor treatment, in addition to anemia, has been shown in murine models of inflammatory bowel disease (IBD). ${ }^{42}$ The number of diseases described for which hydroxylase inhibitors might be a potential treatment option is rapidly expanding. Dr Martin Schneider (University of Heidelberg, Heidelberg, Germany) presented data demonstrating that pharmacologic hydroxylase inhibition leads to enhanced liver regeneration following hepatectomy, which could be beneficial in diseases such as colon cancer, where metastases are often found in the liver, leading to the resection of large portions of the organ. ${ }^{43}$ Dr Sean P Colgan, Dr Yatrik Shah (University of Michigan, Ann Arbor, MI, USA), and Dr Eric L Campbell (University of Colorado, Anschutz Medical Campus, Aurora, CO, USA) made a critical point about the importance of HIF- $1 \alpha$ and HIF- $2 \alpha$ stabilization in IBD, highlighting the potential of targeting the hydroxylases pharmacologically for treatment of IBD. Interestingly, a new delivery mechanism for drugs (including hydroxylase inhibitors) allowing low-dose targeting of specific areas of the gut for treatment of diseases such as IBD was reported by the company Sigmoid Pharma.

Dr Luke AJ O’Neill (Trinity College Dublin, Dublin, Ireland) presented findings that metabolic reprogramming of macrophages is an important factor in immunity and that HIF-1 $\alpha$ plays a critical role in these regulations. Furthermore, he showed that pharmacologic interference with these processes can be anti-inflammatory. Overall, several speakers at this meeting highlighted the anti-inflammatory action of hydroxylase inhibitors and showed the great potential and interest in this mode of action of these drugs.

The importance of oxygen sensing and missing links in the current knowledge of oxygen sensing were highlighted by the keynote speaker, Dr Salvador Moncada (University of Manchester, Manchester, UK). He presented a retrospective of his work, indicating that oxygen and NO interact at the level of the cytochrome oxidase complex IV and that NO might regulate oxygen consumption. He emphasized that this important question needs future studies to further elucidate the interaction between oxygen and $\mathrm{NO}$ in oxygen sensing.

\section{Concluding remarks}

In addition to the oral presentations overviewed in the present short report, there were over 300 posters presented at the Keystone and HypoxiaNet Symposium, which reflected the scale of hypoxia research and represented the broad range of interests within the field. Overall, the symposium allowed the attendees to disseminate new scientific knowledge, to discuss new methodologies, and to form new collaborations. In addition, it also facilitated interaction between clinicians and academic and industrial researchers, and was a major educational platform for junior researchers in the field. The next Keystone Symposium of the hypoxia research field will be held in 2017, with Dr M Celeste Simon as lead organizer. The 2015 symposium ended as any important and intriguing meeting should, with an anthem written and performed by Dr Cormac T Taylor about the field of and the dedication to hypoxia research: https://www.youtube.com/ watch? $\mathrm{v}=\mathrm{nD} 8 \mathrm{gzUOYnfE}$ and https://www.youtube.com/ watch? $\mathrm{v}=\mathrm{RrFe} 4 \mathrm{mEWeNU}$.

\section{Acknowledgments}

We thank the Keystone Symposia for their contribution and generosity in the organization of this meeting. This meeting was held in collaboration with Science Foundation Ireland. We would particularly like to thank the four main organizers of the meeting: Dr Cormac T Taylor (University College Dublin, Dublin, Ireland), Dr M Celeste Simon (HHMI/University of Pennsylvania, Philadelphia, PA, USA), Dr Sean P Colgan (University of Colorado, Denver, CO, USA), and Dr Roland H Wenger (University of Zürich, Zürich, Switzerland). We would also like to acknowledge all researchers in the hypoxia field for the scientific contributions that were made at this symposium. We regret that we were unable to reference all presentations in the current short report. 


\section{Disclosure}

The authors report no conflicts of interest in this work.

\section{References}

1. Keystone Symposia on Molecular and Cellular Biology [homepage on the Internet]. Available from http://www.keystonesymposia.org/. Accessed 28 September, 2015.

2. Semenza GL. Hypoxia-inducible factors in physiology and medicine. Cell. 2012; 148:399-408.

3. Semenza GL. Hypoxia-inducible factors: mediators of cancer progression and targets for cancer therapy. Trends Pharmacol Sci. 2012; 33:207-214.

4. Kempf VA, Lebiedziejewski M, Alitalo K, et al. Activation of hypoxiainducible factor-1 in bacillary angiomatosis: evidence for a role of hypoxia-inducible factor-1 in bacterial infections. Circulation. 2005; 111:1054-1062.

5. Kennedy A, Ng CT, Biniecka M, et al. Angiogenesis and blood vessel stability in inflammatory arthritis. Arthritis Rheum. 2010; 62:711-721.

6. Taylor CT, Colgan SP: Hypoxia and gastrointestinal disease. J Mol Med. 2007; 85:1295-1300.

7. Prabhakar NR, Semenza GL. Oxygen Sensing and Homeostasis. Physiology (Bethesda). 2015; 30(5):340-348.

8. Jaakkola P, Mole DR, Tian YM, et al. Targeting of HIF- $\alpha$ to the von Hippel-Lindau ubiquitylation complex by $\mathrm{O} 2$-regulated prolyl hydroxylation. Science. 2001; 292:468-472.

9. Bruick RK, McKnight SL. A conserved family of prolyl-4-hydroxylases that modify HIF. Science. 2001;294(5545):1337-1340.

10. Epstein AC, Gleadle JM, McNeill LA, et al. C. elegans EGL-9 and mammalian homologs define a family of dioxygenases that regulate HIF by prolyl hydroxylation. Cell. 2001;107(1):43-54.

11. Lando D, Peet DJ, Gorman JJ, Whelan DA, Whitelaw ML, Bruick RK. FIH-1 is an asparaginyl hydroxylase enzyme that regulates the transcriptional activity of hypoxia-inducible factor. Genes Dev. 2002;16(12): 1466-1471.

12. Mahon PC, Hirota K, Semenza GL. FIH-1: a novel protein that interacts with HIF-1alpha and VHL to mediate repression of HIF-1 transcriptional activity. Genes Dev. 2001;15(20):2675-2686.

13. Semenza GL. Hypoxia-inducible factors: mediators of cancer progression and targets for cancer therapy. Trends Pharmacol Sci. 2012; 33:207-214.

14. Fraisl P, Aragones J, Carmeliet P. Inhibition of oxygen sensors as a therapeutic strategy for ischaemic and inflammatory disease. Nat Rev Drug Discov. 2009;8:139-152.

15. Hewitson KS, McNeill LA, Riordan MV, et al. Hypoxia-inducible factor (HIF) asparagine hydroxylase is identical to factor inhibiting HIF (FIH) and is related to the cupin structural family. J Biol Chem. 2002;277(29):26351-26355.

16. Koury MJ, Haase VH. Anaemia in kidney disease: harnessing hypoxia responses for therapy. Nat Rev Nephrol. 2015;11(7):394-410.

17. Semenza GL, Wang GL. A nuclear factor induced by hypoxia via de novo protein synthesis binds to the human erythropoietin gene enhancer at a site required for transcriptional activation. Mol Cell Biol. 1992;12(12):5447-5454.

18. Cummins EP, Berra E, Comerford KM, et al. Prolyl hydroxylase-1 negatively regulates IkappaB kinase-beta, giving insight into hypoxiainduced NFkappaB activity. Proc Natl Acad Sci U S A. 2006;103(48): 18154-18159.

19. Moser SC, Bensaddek D, Ortmann B, et al. PHD1 links cell-cycle progression to oxygen sensing through hydroxylation of the centrosomal protein Cep192. Dev Cell. 2013;26(4):381-392.

20. Zheng X, Zhai B, Koivunen P, et al. Prolyl hydroxylation by EglN2 destabilizes FOXO3a by blocking its interaction with the USP9x deubiquitinase. Genes Dev. 2014;28(13):1429-1444.
21. Scholz CC, Taylor CT. Hydroxylase-dependent regulation of the NF- $\mathrm{KB}$ pathway. Biol Chem. 2013;394(4):479-493.

22. Cockman ME, Lancaster DE, Stolze IP, et al. Posttranslational hydroxylation of ankyrin repeats in IkappaB proteins by the hypoxia-inducible factor (HIF) asparaginyl hydroxylase, factor inhibiting HIF (FIH). Proc Natl Acad Sci U S A. 2006;103(40):14767-14772.

23. Cockman ME, Webb JD, Kramer HB, Kessler BM, Ratcliffe PJ. Proteomics-based identification of novel factor inhibiting hypoxia-inducible factor (FIH) substrates indicates widespread asparaginyl hydroxylation of ankyrin repeat domain-containing proteins. Mol Cell Proteomics. 2009;8(3):535-546.

24. Janke K, Brockmeier U, Kuhlmann K, et al. Factor inhibiting HIF-1 (FIH-1) modulates protein interactions of apoptosis-stimulating p53 binding protein 2 (ASPP2). J Cell Sci. 2013;126(Pt 12):2629-2640.

25. Zheng X, Linke S, Dias JM, et al. Interaction with factor inhibiting HIF-1 defines an additional mode of cross-coupling between the Notch and hypoxia signaling pathways. Proc Natl Acad Sci U SA. 2008;105(9): $3368-3373$.

26. Zhang N, Fu Z, Linke S, et al. The asparaginyl hydroxylase factor inhibiting HIF-1alpha is an essential regulator of metabolism. Cell Metab. 2010;11(5):364-378.

27. Keith B, Johnson RS, Simon MC. HIF $1 \alpha$ and HIF $2 \alpha$ : sibling rivalry in hypoxic tumour growth and progression. Nat Rev Cancer. 2012;12(1): 9-22.

28. Frede S, Stockmann C, Freitag P, Fandrey J. Bacterial lipopolysaccharide induces HIF-1 activation in human monocytes via p44/42 MAPK and NF-kappaB. Biochem J. 2006;396(3):517-527.

29. Rius J, Guma M, Schachtrup C, et al. NF-kappaB links innate immunity to the hypoxic response through transcriptional regulation of HIF1alpha. Nature. 2008;453(7196):807-811.

30. Bonello S, Zähringer C, BelAiba RS, et al. Reactive oxygen species activate the HIF-1alpha promoter via a functional NFkappaB site. Arterioscler Thromb Vasc Biol. 2007;27(4):755-761.

31. Bruning U, Cerone L, Neufeld Z, et al. MicroRNA-155 promotes resolution of hypoxia-inducible factor 1alpha activity during prolonged hypoxia. Mol Cell Biol. 2011;31(19):4087-4096.

32. Semenza GL. Oxygen sensing, hypoxia-inducible factors, and disease pathophysiology. Апnи Rev Pathol. 2014;9:47-71.

33. Semenza GL. Oxygen sensing, homeostasis, and disease. NEngl J Med. 2011;365(6):537-547.

34. Wenger RH, Hoogewijs D. Regulated oxygen sensing by protein hydroxylation in renal erythropoietin-producing cells. Am J Physiol Renal Physiol. 2010;298(6):F1287-F1296.

35. Teppema LJ, Dahan A. The ventilatory response to hypoxia in mammals: mechanisms, measurement, and analysis. Physiol Rev. 2010; 90(2):675-754.

36. López-Barneo J, Ortega-Sáenz P, Pardal R, Pascual A, Piruat JI. Carotid body oxygen sensing. Eur Respir J. 2008;32(5):1386-1398.

37. Ackerman D, Simon MC. Hypoxia, lipids, and cancer: surviving the harsh tumor microenvironment. Trends Cell Biol. 2014;24(8):472-478.

38. Wilson WR, Hay MP. Targeting hypoxia in cancer therapy. Nat Rev Cancer. 2011;11(6):393-410.

39. Majmundar AJ, Wong WJ, Simon MC. Hypoxia-inducible factors and the response to hypoxic stress. Mol Cell. 2010;40(2):294-309.

40. Semenza GL. Targeting HIF-1 for cancer therapy. Nat Rev Cancer. 2003;3(10):721-732.

41. Yan L, Colandrea VJ, Hale JJ. Prolyl hydroxylase domain-containing protein inhibitors as stabilizers of hypoxia-inducible factor: small molecule-based therapeutics for anemia. Expert Opin Ther Pat. 2010; 20(9):1219-1245.

42. Eltzschig HK, Bratton DL, Colgan SP. Targeting hypoxia signalling for the treatment of ischaemic and inflammatory diseases. Nat Rev Drug Discov. 2014;13(11):852-869.

43. Frankel TL, D'Angelica MI. Hepatic resection for colorectal metastases. J Surg Oncol. 2014;109(1):2-7. 
Hypoxia

Dovepress

\section{Publish your work in this journal}

Hypoxia is an international, peer-reviewed, open access journal that aims to improve understanding of the biological response to hypoxia. The journal will publish original research articles, reviews, methodological advances, clinical studies, and expert opinions that identify developments in the regulation of the physiological and pathological responses to The manuscript management system is completely online and includes a very quick and fair peer-review system, which is all easy to use. Visit http://www.dovepress.com/testimonials.php to read real quotes from published authors. 